\title{
Efficient Verification of Multi-Property Designs (The Benefit of Wrong Assumptions)
}

\author{
Eugene Goldberg, Matthias Güdemann, Daniel Kroening \\ Diffblue Ltd. \\ Oxford, UK
}

\author{
Rajdeep Mukherjee \\ University of Oxford \\ UK
}

\begin{abstract}
We consider the problem of efficiently checking a set of safety properties $P_{1}, \ldots, P_{k}$ of one design. We introduce a new approach called $\mathbf{J}_{A}$-verification where $\mathbf{J}_{A}$, stands for "JustAssume" (as opposed to "assume-guarantee"). In this approach, when proving a property $P_{i}$, one assumes that every property $P_{j}$ for $j \neq i$ holds. The process of proving properties either results in showing that $P_{1}, \ldots, P_{k}$ hold without any assumptions or finding a "debugging set" of properties. The latter identifies a subset of failed properties that are the first to break. The design behaviors that cause the properties in the debugging set to fail must be fixed first. Importantly, in our approach, there is no need to prove the assumptions used. We describe the theory behind our approach and report experimental results that demonstrate substantial gains in performance, especially in the cases where a small debugging set exists.
\end{abstract}

\section{INTRODUCTION}

The advent of powerful model checkers based on SAT [1]-[4] has created a new wave of research in property checking. This research has been mostly focused on algorithms that verify a single property for a given design. However, in practice, engineers write many properties for one design (sometimes hundreds and even thousands). This demands efficient and scalable techniques for automatic verification of multiple properties for one design.

More specifically, the problem we address is as follows. We are given a transition relation and a set of initial states, which specify the design. In addition, we are given a set of safety properties $P_{1}, \ldots, P_{k}$ that are expected to hold. (In Section 5, we consider the case where some properties are expected to fail.) We want to check if every property $P_{i}$ holds. If not, we want to have an efficient way to identify failed properties that point to wrong design behaviors. (Thus, identification of all failed properties is not mandatory.) One way to solve this problem is to check whether the property $P:=P_{1} \wedge \ldots \wedge P_{k}$ holds. We will call $P$ the aggregate property. If $P$ holds, then all properties $P_{i}$ are proven. Otherwise, the generated counterexample (CEX for short) identifies a subset of the failed properties, but no information is gained about the remaining properties. The latter can be verified by removing the failed properties from the set, and re-iterating the procedure with a new aggregate property.

In this paper, we study an alternative approach where the properties $P_{i}$ are verified separately. We will refer to this approach as separate verification as opposed to joint verification

Supported by ERC project 280053 "CPROVER", the H2020 FET OPEN 712689 SC $^{2}$ and SRC contracts no. 2012-TJ-2269 and 2016-CT-2707. of a set of properties. We will highlight three main reasons for our interest in separate verification. First, we want to study multi-property verification in the context of an IC3like model checker [3]. Such a model checker will benefit from separate verification by generating proofs that take into account the specifics of each property. Besides, a property $P_{i}$ is a weaker version of the aggregate property $P$. Thus, proving $P_{i}$ should be easier than $P$. Second, each property $P_{i}$ is an over-approximation of the same set of reachable states. Therefore, inductive invariants of already proven properties can be re-used. The third reason is as follows. If all $P_{i}$ are true, there is a common proof of this fact, namely a proof that $P$ holds. However, if some $P_{i}$ properties fail, there may not be one universal CEX that explains all failures. Moreover, in many cases, failures are specific to one property and therefore separate verification is more relevant in such a context.

In this paper, we introduce a version of separate verification called JA-verification. Here, JA stands for "Just-Assume", as opposed to "assume-guarantee". In JA-verification, one proves property $P_{i}$, assuming that every other property $P_{j}$, for $j \neq i$, holds, regardless whether it is true. We will call such a proof local as opposed to a global proof that $P_{i}$ is true where no assumptions are made. JA-verification results in constructing the set of properties $P_{i}$ that failed locally (if any). We show that if the aggregate property $P$ fails, there is at least one property $P_{i}$ that fails both globally and locally. Thus, if all properties $P_{i}$ hold locally it also means they hold globally.

If $P_{i}$ holds locally, it either holds globally as well or every CEX that breaks $P_{i}$ fails some other property $P_{j}$ before this CEX fails $P_{i}$. That is, a CEX for $P_{i}$ contains a shorter CEX for $P_{j}$. This suggests that if $P_{i}$ holds locally, its failure (if any) is most likely caused by failures of other properties. For this reason, we call the set of properties that fail locally a debugging set. This debugging set of properties points to design behaviors that need to be fixed in the first place. The approach guarantees that the failure of a property from the debugging set is not preceded by a failure of any other property. JA-verification constructs a debugging set as follows: when proving $P_{i}$ it assumes that $P_{j}$, for $j \neq i$, hold even when some of them fail. Thus, even the wrong assumption that all $P_{j}, j \neq i$ hold proves to be useful. For that reason, we use the term "Just Assume" to name our approach.

To improve the efficiency of JA-verification, we exploit the fact that IC3 proves a property by strengthening it to make this 
property inductive. Specifically, we show that the strengthening clauses generated by Ic 3 can be re-used when making any other property inductive if the same transition relation and initial states are used. Thus, in JA-verification, clauses generated to make $P_{i}$ inductive are re-used when proving $P_{j}, j \neq i$.

Our contribution is threefold. First, we describe a new method of multi-property verification called JA-verification (see Section 4). It is based on the machinery of local proofs (Sections 2 and 3) and re-use of strengthening clauses (Section 6). Second, we show that JA-verification generates CEXs only for a special subset of failed properties called a debugging set. This is very important since computing a CEX can be very expensive (e.g., if a counter is involved). Third, we provide implementation details (Section 7) and experimental results showing the viability of JA-verification (Section 8).

\section{Local And Global Proofs}

Separate verification is based on the assumption that, in general, proving $P_{i}$ is easier than $P_{1} \wedge \ldots \wedge P_{k}$ because $P_{i}$ is a weaker property. In this section, we discuss how one can make a proof of a property simpler if this proof is only needed in the context of proving a stronger property.

\section{A. Definitions}

We will denote the predicates of the transition relation and the initial states as $T\left(S, S^{\prime}\right)$ and $I(S)$ respectively. Here $S$ and $S^{\prime}$ are sets of present and next state variables respectively. An assignment $s$ to variables $S$ is called a state. We will refer to a state satisfying a predicate $Q(S)$ as a $Q$-state. A property is just a predicate $P(S)$. We will say that a $P$-state (respectively $\bar{P}$-state) is a good (respectively $b a d$ ) state. A property $P$ is called inductive with respect to $T$ if $P(S) \wedge T \rightarrow P\left(S^{\prime}\right)$ holds.

We will call a sequence of states $\left(s_{j}, \ldots, s_{k}\right)$ a trace if $T\left(s_{i}, s_{i+1}\right)$ is true for $i=j, \ldots, k-1$. We will call the trace above initialized if $s_{j}$ is an $I$-state. Given a property $P(S)$ where $I \rightarrow P$, a CEX is an initialized trace $\left(s_{0}, \ldots, s_{k}\right)$ where $s_{i}$, $i=0, \ldots, k-1$ are $P$-states and $s_{k}$ is a $\bar{P}$-state. We will refer to a state transition system with initial states $I$ and transition relation $T$ as an $(I, T)$-system. Given an $(I, T)$-system, checking a property $P$ is to find a CEX for $P$ or to show that none exists.

\section{B. Hardness of proving strong and weak properties}

Let $P$ and $Q$ be two properties where $Q$ is weaker than $P$, i.e., $P \rightarrow Q$. On the one hand, verification of $Q$ should be easier because one needs to prove unreachability of a smaller set of bad states. On the other hand, $P$ can be inductive even if $Q$ is not. In fact, the essence of IC3 is to turn a non-inductive property into an inductive one by adding strengthening clauses. This makes the modified property easier to prove despite the fact that it is stronger from a logical point of view.

The reason for the paradox above is as follows. The set of traces one needs to consider to prove $Q$ is not a subset of those one considers when proving $P$. To prove $P$, one needs to show that there is no initialized trace of $P$-states leading to a $\bar{P}$-state. Thus, one does not consider traces where two $\bar{P}$-states occur. Proving $Q$ is reduced to showing that there is no initialized trace of $Q$-states leading to a $\bar{Q}$-state. Since $P \rightarrow Q$, a $\bar{Q}$-state is a $\bar{P}$-state as well. On the other hand, a $Q$-state can also be a $\bar{P}$-state. Thus, in contrast to the case when we prove $P$, to prove $Q$ one has to consider traces that may include two or more different $\bar{P}$-states.

We will refer to a regular proof of $Q$ (where one shows that no initialized trace of $Q$-states leads to a $\bar{Q}$-state) as a global one. In the next subsection, we discuss reducing the complexity of proving $Q$ using the machinery of local proofs.

\section{Local proofs}

The intuition behind local proofs is as follows. Suppose that one needs to prove a property $Q$ as a step in proving a stronger property $P$. Then it is reasonable to ignore traces that do not make sense from the viewpoint of proving $P$. Proving $Q$ locally in the context of $P$, or just locally for short, is to show that there does not exist an initialized trace of $P$-states (rather than $Q$-states) leading to a $\bar{Q}$-state.

The importance of local proofs is twofold. First, to prove $Q$ locally, one needs to consider only a subset of the traces to prove $P$ (because the set of $\bar{Q}$-states is a subset of that of $\bar{P}$-states). Thus, in terms of the set of traces to consider, a weaker property becomes also "easier". Second, as we show in Section 4, to prove the aggregate property $P:=P_{1} \wedge \ldots \wedge P_{k}$, it suffices to prove all properties $P_{i}$ locally with respect to $P$.

It is convenient to formulate the notion of a local proof in terms of a modified transition relation $T$. We will call this modification the projection of $T$ onto property $P$ and denote it as $T^{P}$. It is defined as follows.

- $T^{P}\left(s, s^{\prime}\right)=T\left(s, s^{\prime}\right)$, if $s$ is a $P$-state.

- $T^{P}\left(s, s^{\prime}\right)=0$ if $s$ is a $\bar{P}$-state and $s \neq s^{\prime}$.

- $T^{P}\left(s, s^{\prime}\right)=1$ if $s$ is a $\bar{P}$-state and $s=s^{\prime}$.

Informally, $T^{P}$ is obtained from $T$ by excluding any transitions from a $\bar{P}$-state other than a transition to itself. Hence, a trace in $\left(I, T^{P}\right)$-system cannot have two different $\bar{P}$-states. Thus, a local proof of $Q$ with respect to property $P$, as we introduced above, is just a regular proof with respect to $T^{P}$. (In turn, proving $Q$ globally is done with respect to $T$.)

Proposition 1: Let $P$ be inductive with respect to transition relation $T$. Then any property $Q$ weaker than $P$ (i.e., $P \rightarrow Q$ ) is inductive with respect to $T^{P}$.

(The proofs of the propositions are given in [5].) Proposition 1 states that in terms of proofs by induction, proving $Q$ locally with respect to a stronger property $P$ is at most as hard as proving $P$ itself.

\section{Local Proofs And Debugging}

In this section, we explain how the machinery of local proofs can be used to address the following problem. Given a property $P$ that failed, find a weaker property $Q$ that is false as well and can be viewed as an explanation for failure of $P$. The subtlety here is that not every failed property $Q$ where $P \rightarrow Q$ can be viewed as a reason for why $P$ fails. We will refer to this problem as the debugging problem.

To address the debugging problem one first needs to clarify the relation between local and global proofs. 
Proposition 2: Let $P \rightarrow Q$. If property $Q$ holds with respect to $T$ (i.e., globally), it also holds with respect to $T^{P}$ (i.e., locally). The opposite is not true. If $Q$ holds with respect to $T^{P}$, it either holds with respect to $T$ or it fails with respect to $T$ and every CEX contains at least two $\bar{P}$-states $s^{\prime}$ and $s^{\prime \prime}$ where $s^{\prime} \neq s^{\prime \prime}$.

Informally, Proposition 2 means that proving $Q$ locally is almost "as good as" proving globally modulo CEXs that do not make sense from the viewpoint of proving $P$. These CEXs have at least two $\bar{P}$-states.

One can use Proposition 2 for solving the debugging problem as follows. Suppose that $Q$ does not hold locally. This means that there is a CEX of $P$-states leading to a $\bar{Q}$-state. Since $P \rightarrow Q$, a $\bar{Q}$-state is a $\bar{P}$-state as well. So this CEX is also a regular CEX for $P$. In other words, the fact that $Q$ fails locally means that $Q$ can be viewed as a reason for failure of $P$.

Suppose that $Q$ holds locally. Assume that $Q$ fails globally and $\left(s_{0}, \ldots, s_{m}\right)$ is a CEX where $s_{m}$ is a $\bar{Q}$-state. From Proposition 2, it follows that this CEX has at least two $\bar{P}$-states. One of these states is $s_{m}$ (because $P \rightarrow Q$ ). Another $\bar{P}$-state is one of $Q$-states $s_{i}, i=1, \ldots, m-1$. This means that failure of $Q$ is not a reason for failure of $P$. Indeed, in every CEX for $Q$, property $P$ fails before $Q$ does.

Summarizing, if property $Q$ fails (respectively holds) locally with respect to $P$, failure of $Q$ is a reason (respectively cannot be a reason) for failure of $P$.

\section{JA-VERIFICATION}

In this section, we present a version of separate verification called "Just-Assume" or JA-verification. As before, $P$ denotes the aggregate property $P_{1} \wedge \ldots \wedge P_{k}$ and $T^{P}$ denotes the projection of $T$ onto $P$ (see Subsection 2-C). Since every property $P_{i}$ is a weaker version of $P$, one can use the results of Sections 2 and 3 based on the machinery of local proofs.

We now provide a justification of proving weaker properties locally in the context of multi-property verification. By using transition relation $T^{P}$ to prove $P_{i}$, one essentially assumes that every property $P_{j}, j \neq i$ holds. While this may not be the case, nevertheless it works for two reasons. The first reason is that if the aggregate property $P$ fails, there is a time frame where $P$ (and hence some property $P_{i}$ ) fails for the first time. Let this be time frame number $m$. For every time frame number $p$ where $p<m$, the assumption that every property $P_{j}, j \neq i$ holds is true. Thus, if $P$ fails, there is at least one property (in our case $P_{i}$ ) that fails even with respect to $T^{P}$.

Here is the second reason why assuming $P_{j}, j \neq i$ works. To get some debugging information when proving property $P_{i}$, one is interested in traces where $P_{i}$ fails before any other property does. By assuming $P_{j}, j \neq i$ is true, one drops the traces where $P_{i}$ fails after some $P_{j}, j \neq i$ has failed.

The propositions below formalize the relation between local proofs and multi-property verification.

Proposition 3: Property $P$ holds with respect to $T$ iff every $P_{i}, i=1, \ldots, k$ holds with respect to $T$.

Proposition 4: Property $P$ holds with respect to $T$ iff $P$ holds with respect to $T^{P}$.
Taking into account that Proposition 3 can also be formulated in terms of $T^{P}$ (instead of $T$ ), Propositions 3 and 4 entail the proposition below.

Proposition 5: Property $P$ holds with respect to $T$ iff every $P_{i}, i=1, \ldots, k$ holds with respect $T^{P}$.

We will refer to the subset of $\left\{P_{1}, \ldots, P_{k}\right\}$ that consist of properties that fail with respect to $T^{P}$, i.e., locally as a debugging set. The following proposition justifies this name.

Proposition 6: Let the aggregate property $P$ fail. Let $D$ denote the debugging set of properties. Then the failure of properties of $D$ is the reason for the failure of $P$ in the following sense. For each CEX $\left(s_{0}, \ldots, s_{m}\right)$ for property $P$, the state $s_{m}$ that falsifies $P$ also falsifies at least one property $P_{i} \in D$.

Example 1: The Verilog code below gives an example of an 8-bit counter. This counter increments its value every time the enable signal is true. Once the counter reaches the value of rval it resets its value to 0 . We also want to reset the counter when signal req is true (regardless of the current value of the counter). However, the code contains a buggy line (marked in blue), which prohibits a reset only unless req is true.

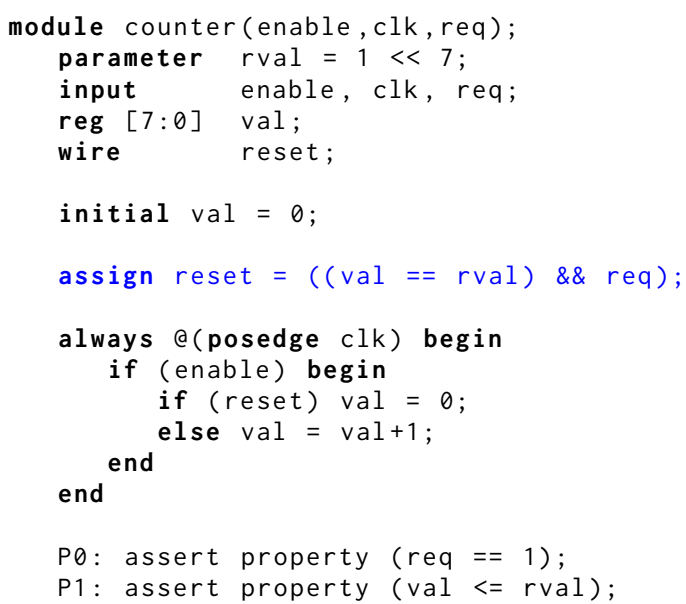

\section{endmodule}

Let us consider verification of properties $P_{0}$ and $P_{1}$ specified by the last two lines of the module counter. Property $P_{0}$ fails globally in every time frame because req is an input variable taking values 0 and 1 . Property $P_{1}$ fails globally due to the bug above. Note however, that only property $P_{0}$ fails locally. Indeed, $P_{0}$ fails even under assumption $P_{1} \equiv 1$. However, $P_{1}$ becomes true if one assumes $P_{0} \equiv 1$. The latter means that $r e q \equiv 1$ and so the counter always resets on reaching value rval. So the debugging set consists only of $P_{0}$. The fact that $P_{1}$ holds locally means that either $P_{1}$ is true globally or that any CEX failing $P_{1}$ first fails $P_{0}$. The latter implies that the failure of $P_{1}$ is caused by incorrect handling of variable req.

Note that proving $P_{1}$ false globally is hard for a large counter because a CEX consists of all states of the counter from 0 to rval. On the contrary, proving $P_{1}$ true under assumption $P_{0} \equiv 1$ is trivial because $P_{1}$ is inductive under this assumption. In Table I, we compare proving properties $P_{0}$ and $P_{1}$ above globally and locally. The first column gives the size of the counter. The next four columns give the results of solving 
TABLE I

Example with a counter. Time limit is 1 hour

\begin{tabular}{|r|r|r|r|r|r|}
\hline \#bits & \multicolumn{4}{|c|}{ solving globally } & \multirow{2}{*}{$\begin{array}{l}\text { solving } \\
\text { locally }\end{array}$} \\
\cline { 2 - 5 } & \multicolumn{2}{|c|}{ ABC (bmc) } & \multicolumn{2}{c|}{ ABC (pdr) } & \\
\cline { 2 - 5 } & \#time frames & time & \#time frames & time & \\
\hline 8 & 128 & $0.3 \mathrm{~s}$ & 10 & $0.1 \mathrm{~s}$ & $0.01 \mathrm{~s}$ \\
\hline 12 & 2,048 & $723 \mathrm{~s}$ & 51 & $1.7 \mathrm{~s}$ & $0.02 \mathrm{~s}$ \\
\hline 14 & $*$ & $*$ & 118 & $9.9 \mathrm{~s}$ & $0.02 \mathrm{~s}$ \\
\hline 16 & $*$ & $*$ & 269 & $113 \mathrm{~s}$ & $0.02 \mathrm{~s}$ \\
\hline 18 & $*$ & $*$ & 315 & $1,278 \mathrm{~s}$ & $0.02 \mathrm{~s}$ \\
\hline 20 & $*$ & $*$ & $*$ & $*$ & $0.02 \mathrm{~s}$ \\
\hline
\end{tabular}

$P_{0}$ and $P_{1}$ globally by $\mathrm{ABC}$, a mature tool developed at UC Berkeley [6]. The first pair of columns gives the results of Bounded Model Checking [1] (the largest number of used time frames and run time). The next pair of columns provides results of PDR (i.e., IC3). Finally, we give the results of solving $P_{0}$ and $P_{1}$ locally by our tool (see Section 7).

The results show that bounded model checking soon becomes impractical, as the number of time frames increases exponentially. ABC's PDR solves more cases, but to generate a CEX, it has to consider a quickly increasing number of time frames as well. For JA-verification, the size of the counter has no influence on the run time. While the counter is a purely synthetic example, in practice, one often has to find so-called deep counterexamples. A system with complex inner state might require a long sequence of steps to reach a buggy state.

\section{Handling Properties Expected To Fail}

When proving properties $P_{i}, i=1, \ldots, k$ in JA-verification, as introduced in Section 4, one excludes the traces where a property $P_{j}, j \neq i$ fails before $P_{i}$ does. This is based on the assumption that the properties that are the first to fail indicate design behaviors that need to be fixed first. However, this assumption is unreasonable when a property $P_{j}$ that fails before $P_{i}$ is Expected To Fail (ETF). For instance, to ensure that a state $s$ is reachable, one may formulate an ETF property $P_{j}$ where $s$ is a $\bar{P}_{j}$-state. In this case, excluding the traces where $P_{j}$ fails before $P_{i}$ is a mistake.

One can easily extend JA-verification to handle ETF properties as follows. Suppose that our objective is to prove every property $P_{i}$ that is Expected To Hold (ЕTH). In addition, for every ETF property we want to find a CEX that does not break any Етн property. Then, to solve $P_{i}, i=1, \ldots, k$ locally one assumes that every ETH $P_{j}, j \neq i$ is true. Thus, we exclude the traces where Етн properties fail before $P_{i}$, even if the latter is an ETF property.

\section{IC3 And Clause Re-using}

So far, we discussed the machinery of local proofs without specifying the algorithm used to prove a property. In this section, we describe an optimization technique applicable if property checking is performed by Ic3 [3]. The essence of this technique is to re-use strengthening clauses generated by IC 3 for property $P_{i}$ to strengthen another property $P_{j}, j \neq i$.

\section{A. Brief description of IC3}

Let $Q$ be a property of an $(I, T)$-system where $I \rightarrow Q$. If $Q$ holds, there always exists a predicate $F(S)$ such that $Q \wedge F$ is inductive with respect to $T$. Then $(Q \wedge F \wedge T) \rightarrow\left(Q^{\prime} \wedge F^{\prime}\right)$, where a primed predicate symbol means that the predicate in question depends on next state variables $S^{\prime}$ (for instance, $Q^{\prime}$ denotes $\left.Q\left(S^{\prime}\right)\right)$. The fact that $Q \wedge F$ is inductive implies that $Q \wedge F$ is an over-approximation of the set of states reachable in the $(I, T)$ - system in question. Therefore, for every state $s$ reachable in $(I, T)$-system, $Q(s) \wedge F(s)=1$.

In IC3, formulas are represented in conjunctive normal form and the predicate $F$ is constructed as a set of clauses (disjunctions of literals). Let $R \operatorname{ch}(I, T, j)$ denote the set of states reachable from $I$-states in at most $j$ transitions. To construct the formula $F$, IC 3 builds a sequence of formulas $F_{0}, \ldots, F_{m}$ where $F_{0}=I$ and $F_{j}, j=1, \ldots, m$ specifies an over-approximation of $\operatorname{Rch}(I, T, j)$. That is, if a state $s$ is in $\operatorname{Rch}(I, T, j)$, then $s$ satisfies $F_{j}$, i.e., $F_{j}(s)=1$. A formula $F_{j}, j>0$ is initialized with $Q$. Then $F_{j}$ (and possibly some formulas $F_{i}, i<j$ ) are strengthened by adding so called inductive clauses. If $Q \wedge F_{i}$ becomes inductive for some value of $i, i \leq j$, property $Q$ holds. Clause $C$ is called inductive relative to $F_{j}$ if $I \rightarrow C$ and $C \wedge F_{j} \wedge T \rightarrow C^{\prime}$ hold. In this case, every $s \in R \operatorname{ch}(I, T, j)$ satisfies $C$ and so $F_{j} \wedge C$ is still an over-approximation of $\operatorname{Rch}(I, T, j)$.

\section{B. Re-using strengthening clauses}

The idea of re-using strengthening clauses is based on the following observation. Suppose that $F_{Q}$ is a set of clauses which makes $Q$ inductive in the $(I, T)$-system. This means that $Q \wedge F_{Q}$ is an over-approximation of the set of all states reachable in the $(I, T)$-system. Hence a state $s \in R \operatorname{ch}(I, T, j)$ satisfies $F_{Q}$, for any value $j \geq 0$. Suppose one needs to prove some other property $R$. Then, when constructing a formula over-approximating $\operatorname{Rch}(I, T, j)$, one can initialize this formula with $R \wedge F_{Q}$, rather than with $R$.

\section{IMPLEMENTATION}

We use a version of IC3 developed in our research group. We will refer to it as $I c 3-d b$ where "db" stands for Diffblue. We will refer to our implementation of JA-verification based on Ic3-db as Ja-ver. The latter is a Perl script, which calls $I c 3-d b$ in a loop for proving individual properties.

Let $P_{1}, \ldots, P_{k}$ be the set of properties to be proved. Proving $P_{i}$ locally means showing that there is no initialized trace of $P$ states that leads to a $\overline{P_{i}}$-state, where $P$ is the aggregate property $P_{1} \wedge \ldots \wedge P_{k}$. To guarantee that all present states satisfy $P, I c 3-d b$ adds constraints to the transition relation $T$ that force $P_{j}, j \neq i$ to be equal to 1 . Adding constraints, in general, adversely affects an important optimization technique of Ic3 called state lifting [4], [7]. For that reason, Ic3-db has an option to make the state lifting procedure ignore constraints specified by $P_{j}$, $j \neq i$. The interaction of state lifting and property constraints in $I c 3-d b$ is described in more detail in a technical report [5]. This report also provides more information on how re-use of strengthening clauses is implemented in Ic3- $d b$.

\section{EXPERIMENTAL RESUltS}

In this section, we report experiments that show the viability of our approach to multi-property verification. We use 
TABLE II

Designs with failed properties. Many properties of $6 s 258,6 s 207,6 s 254$, $6 s 335$, and $6 s 380$ are false globally in joint verification but true locally in JA-verification. 'mem' means running out of memory

\begin{tabular}{|c|c|c|c|c|c|c|c|c|c|}
\hline \multirow[t]{3}{*}{ name } & \multirow[t]{3}{*}{ \#latch } & \multirow{3}{*}{$\begin{array}{l}\text { \#pro } \\
\text { pert. }\end{array}$} & \multicolumn{4}{|c|}{ Joint verification } & \multirow{2}{*}{\multicolumn{3}{|c|}{$\begin{array}{c}\text { JA-verification by } I c 3-d b \\
\text { with clause re-use }\end{array}$}} \\
\hline & & & \multicolumn{2}{|c|}{$\overline{\mathrm{ABC}}$} & \multicolumn{2}{|c|}{$I c 3-d b$} & & & \\
\hline & & & $\begin{array}{l}\text { \#false } \\
\text { (\#true) }\end{array}$ & time & $\begin{array}{l}\text { \#false } \\
\text { (\#true) }\end{array}$ & time & $\begin{array}{l}\text { time } \\
\text { limit }\end{array}$ & $\begin{array}{l}\text { \#false } \\
\text { (\#true) }\end{array}$ & $\begin{array}{l}\text { total } \\
\text { time }\end{array}$ \\
\hline $6 s 104$ & 84,925 & 124 & $1(0)$ & $10 \mathrm{~h}$ & $1(0)$ & mem & $0.3 \mathrm{~h}$ & 1 & 2.51 \\
\hline $6 s 260$ & 2,179 & 35 & $1(0)$ & $10 \mathrm{~h}$ & $1(0)$ & $10 \mathrm{~h}$ & $0.5 \mathrm{~h}$ & $1(34)$ & $1,686 \mathrm{~s}$ \\
\hline $6 \mathrm{~s} 258$ & 1,790 & 80 & $25(0)$ & $10 \mathrm{~h}$ & $30(0)$ & $10 \mathrm{~h}$ & $0.3 \mathrm{~h}$ & $1(72)$ & $2.4 \mathrm{~h}$ \\
\hline $6 s 175$ & 7, & 3 & $2(0)$ & $10 \mathrm{~h}$ & $2(0)$ & $10 \mathrm{~h}$ & $0.3 \mathrm{~h}$ & $2(1)$ & $554 \mathrm{~s}$ \\
\hline $6 s 207$ & 3,012 & 33 & $6(0)$ & $10 \mathrm{~h}$ & $10(0)$ & $10 \mathrm{~h}$ & $0.3 \mathrm{~h}$ & $2(31)$ & $22 \mathrm{~s}$ \\
\hline $6 s 254$ & 762 & 14 & $13(1)$ & $25 \mathrm{~s}$ & $13(1)$ & $225 \mathrm{~s}$ & $0.3 \mathrm{~h}$ & $1(13)$ & $2 \mathrm{~s}$ \\
\hline $6 \mathrm{~s} 335$ & 1,658 & 61 & $26(35)$ & $2 \mathrm{~h}$ & $26(35)$ & $260 \mathrm{~s}$ & $0.3 \mathrm{~h}$ & $20(41)$ & $56 \mathrm{~s}$ \\
\hline $6 \mathrm{~s} 380$ & 5,606 & 897 & $399(0)$ & $10 \mathrm{~h}$ & $395(0)$ & $10 \mathrm{~h}$ & $0.3 \mathrm{~h}$ & $3(894)$ & $550 \mathrm{~s}$ \\
\hline
\end{tabular}

benchmarks from the multi-property track of the HwMCC-12 and 13 competitions. We did two experiments, comparing joint and JA-verification based on Ic3- $d b$. For both experiments, we picked eight designs to illustrate the point described in the corresponding subsection. (More experimental data can be found in [5].) We cross-check the results given by Ic3- $d b$ in joint verification with those reported by the latest version of $\mathrm{ABC}[6]$.

As we mentioned in Section 7, JA-verification is implemented as a Perl script $J a$-ver that calls $I c 3-d b$ to process individual properties sequentially. In this paper, we do not exploit the possibility to improve JA-verification by processing properties in a particular order. ${ }^{1}$ Properties are verified in the order they are given in the design description.

Joint verification is also implemented as a Perl script called Jnt-ver, where Ic3- $d b$ is called to verify the aggregate property $P:=P_{1} \wedge \ldots \wedge P_{k}$. If $P$ fails, the individual properties refuted by the generated CEX are reported false. Jnt-ver forms a new aggregate property by conjoining the properties $P_{i}$ that are unsolved yet and calls $I c 3-d b$ again. This continues until every property is solved. As for $\mathrm{ABC}$, joint verification is its natural mode of operation. However, in contrast to Jnt-ver, ABC does not re-start when a property is proved false and goes on with solving the remaining properties.

In both experiments, the time limit for joint verification was set to 10 hours. The time limit used by $I c 3-d b$ in $\mathrm{JA}$-verification to prove one property is indicated in Tables II, III and IV. If a property of a benchmark was not solved by $I c 3-d b$, the time limit was added to the total time of solving this benchmark.

\section{A. Designs with failing properties}

In the first experiment, we picked eight designs with failing properties to show that solving properties locally can be much more efficient than globally. Thus, the sacrifice one makes by looking only for the failed properties that form the debugging set pays off. Unfortunately, the HwMCC competitions do not identify properties of multi-property benchmarks that are expected to fail, if any (see Section 5). For that reason, we assumed that every property was expected to hold.

\footnotetext{
${ }^{1} \mathrm{~A}$ rule of thumb here is to verify easier properties first to accumulate strengthening clauses and use them later for harder properties.
}

TABLE III

All properties are true

\begin{tabular}{|c|c|c|c|c|c|c|c|}
\hline \multirow[t]{2}{*}{ name } & \multirow[t]{2}{*}{ \#latch } & \multirow[t]{2}{*}{$\begin{array}{l}\text { \#pro- } \\
\text { pert. }\end{array}$} & \multicolumn{2}{|c|}{ Joint verification } & \multicolumn{3}{|c|}{$\begin{array}{c}\text { JA-verification by } I c 3-d b \\
\text { with clause re-use }\end{array}$} \\
\hline & & & $\mathrm{ABC}$ & $I c 3-d b$ & $\begin{array}{l}\text { time } \\
\text { limit }\end{array}$ & $\begin{array}{l}\text { \#un- } \\
\text { solved }\end{array}$ & $I c 3-d b$ \\
\hline $6 s 124$ & 6,748 & 630 & $>10 \mathrm{~h}$ & $2.9 \mathrm{~h}$ & $0.8 \mathrm{~h}$ & 0 & $1.9 \mathrm{~h}$ \\
\hline $6 s 135$ & 2,307 & 340 & $123 \mathrm{~s}$ & $335 \mathrm{~s}$ & $0.8 \mathrm{~h}$ & 0 & $746 s$ \\
\hline $6 s 139$ & 16,230 & 120 & $4.7 \mathrm{~h}$ & $1.7 \mathrm{~h}$ & $2.8 \mathrm{~h}$ & 2 & $6.5 \mathrm{~h}$ \\
\hline $6 s 256$ & 3,141 & 5 & $>10 \mathrm{~h}$ & $602 \mathrm{~s}$ & $2.8 \mathrm{~h}$ & 1 & $2.9 \mathrm{~h}$ \\
\hline bob12m09 & 285 & 85 & $1,692 \mathrm{~s}$ & $930 \mathrm{~s}$ & $0.8 \mathrm{~h}$ & 0 & $784 s$ \\
\hline $6 s 407$ & 11,379 & 371 & $1.3 \mathrm{~h}$ & $3.4 \mathrm{~h}$ & $0.8 \mathrm{~h}$ & 0 & $2,077 \mathrm{~s}$ \\
\hline $6 s 273$ & 15,544 & 42 & $1.8 \mathrm{~s}$ & $325 \mathrm{~s}$ & $0.8 \mathrm{~h}$ & 0 & $290 \mathrm{~s}$ \\
\hline $6 s 275$ & 3,196 & 673 & $334 \mathrm{~s}$ & $1,154 \mathrm{~s}$ & $0.8 \mathrm{~h}$ & 0 & $1,611 \mathrm{~s}$ \\
\hline
\end{tabular}

The results are given in Table II. The first column provides the name of the benchmark. The second and third columns give the number of latches and properties, respectively. The next two pairs of columns provide the results of joint verification performed by $\mathrm{ABC}$ and $I c 3-d b$. The first column of the pair gives the number of false and true properties that $\mathrm{ABC}$ or $I c 3-d b$ managed to solve within the time limit. The second column of the pair reports the amount of time taken by $\mathrm{ABC}$ or $I c 3-d b$. The last three columns report data about JA-verification: the time limit per property, the number of false and true properties solved within the time limit, and the total time taken by $I c 3-d b$. In all tables, the run times that do not exceed one hour are given in seconds.

For all examples but $6 s 258$, JA-verification solved all properties locally. On the other hand, for many examples, in joint verification, only a small fraction of properties were solved by $I c 3-d b$ and ABC globally. Let us consider example $6 s 207$ in more detail. JA-verification solved all properties of $6 \$ 207$ fairly quickly generating the debugging set of two properties. On the other hand, joint verification by $I c 3-d b$ proved that ten properties failed globally within 10 hours. Since JA-verification established that only two properties failed locally, eight out of those ten failed properties were true locally. Let $P_{i}$ be one of those eight properties. The CEX found for $P_{i}$ by joint verification first falsifies a property of the debugging set. Thus, we do not know if there is a CEX where $P_{i}$ fails before other properties. JA-verification does not determine whether $P_{i}$ fails but guarantees that every CEX for $P_{i}$ (if any) first fails some other property.

\section{B. Designs where all properties hold}

In the second experiment, we used designs where all properties were true. Recall that if all properties hold locally, they all hold globally as well. Ideally, we would like to use designs with as many properties as possible. However, for such designs, joint verification usually badly loses to JA-verification since the presence of even a few hard properties $P_{i}$ cripples its performance. This problem can be addressed by partitioning $P_{1}, \ldots, P_{k}$ into smaller clusters of properties [8], which is beyond the scope of this paper. Thus, we picked eight designs that have less than a thousand properties and that can be solved by joint verification without partitioning into clusters. The point we are making is that separate verification is competitive with joint verification even for the benchmarks that favor the latter. 
TABLE IV

Re-using strengthening clauses in JA-verification. Time limit per property is the same as in Table III. Verification of 6s124, 6s139, and 6s407 (without clause re-use) was aborted after 10 hours

\begin{tabular}{|l|r|c|r|c|r|}
\hline \multirow{2}{*}{ name } & \multirow{2}{*}{ \#properties } & \multicolumn{2}{|c|}{ without clause re-use } & \multicolumn{2}{c|}{ with clause re-use } \\
\cline { 3 - 6 } & & \#unsolved & time & \#unsolved & time \\
\hline 6s124 & 630 & 505 & $10 \mathrm{~h}$ & 0 & $\mathbf{1 . 9 h}$ \\
\hline 6s135 & 340 & 0 & $2.7 \mathrm{~h}$ & 0 & $\mathbf{7 4 6} \mathbf{~}$ \\
\hline 6s139 & 120 & 116 & $10 \mathrm{~h}$ & 2 & $\mathbf{6 . 5} \mathbf{~ h}$ \\
\hline 6s256 & 5 & 0 & $\mathbf{8 9 2} \mathrm{s}$ & 1 & $2.9 \mathrm{~h}$ \\
\hline bob12m09 & 85 & 0 & $1.1 \mathrm{~h}$ & 0 & $\mathbf{7 8 4} \mathbf{s}$ \\
\hline 6s407 & 317 & 270 & $10 \mathrm{~h}$ & 0 & $\mathbf{2 , 0 7 7} \mathbf{s}$ \\
\hline 6s273 & 42 & 0 & $1,445 \mathrm{~s}$ & 0 & $\mathbf{2 9 0} \mathrm{s}$ \\
\hline 6s275 & 673 & 0 & $3,273 \mathrm{~s}$ & 0 & $\mathbf{1 , 6 1 1} \mathbf{s}$ \\
\hline
\end{tabular}

The results are given in Table III. The first three columns are the same as in Table II. The next two columns give run times of $\mathrm{ABC}$ and $I c 3-d b$ in joint verification. The last three columns provide information about JA-verification: time limit per property, number of unsolved properties and total run time. The best of the run times obtained in joint verification and $\mathrm{JA}$-verification based on $I c 3-d b$ is given in bold. In three cases, joint verification based on $\mathrm{ABC}$ was the fastest but we needed a comparison that uses a uniform setup.

Table III shows that joint verification performed slightly better. In particular, for benchmarks $6 s 139$ and $6 s 256$, JAverification failed to solve some properties with the time limit of 2.8 hours. However, when we verified properties in an order different from the one of design description, both benchmarks were solved in time comparable with joint verification.

To illustrate the benefit of re-using strengthening clauses, Table IV compares JA-verification with and without re-using strengthening clauses on the examples of Table III. Table IV shows that JA-verification with re-using strengthening clauses significantly outperforms its counterpart. The only exception is $6 s 256$, which has only five properties to check.

\section{RELATED WORK}

We found only a few references to research on multi-property verification. In [9], some modifications of $\mathrm{ABC}$ are presented that let it handle multi-property designs. In [8], [10], the idea of grouping similar properties and solving them together is introduced. The similarity of properties is decided based on design structure (e.g., properties with similar cones of influence are considered similar). The main difference of this approach from ours is that the latter is purely semantic. Thus, the optimizations of separate verification we consider (local proofs and re-using strengthening clauses) can be incorporated in any structure-aware approach. One further difference is that the idea of grouping favors correct designs. Grouping may not work well for designs with broken properties that fail for different reasons and thus have vastly different CEXs.

Assume-guarantee reasoning is an important method for compositional verification [11], [12]. It reduces verification of the whole system to checking properties of its components under some assumptions. To guarantee the correctness of verification one needs to prove these assumptions true. As we mentioned earlier, JA-verification uses yet-unproven properties as assumptions without subsequent justification. This is achieved by our particular formulation of multi-property verification. Instead of proving or refuting every property, JAverification builds a subset of failed properties that are the first to break or proves that this subset is empty.

\section{CONCLusions}

We consider the problem of verifying multiple properties $P_{1}, \ldots, P_{k}$ of the same design. We make a case for separate verification where properties are proved one by one as opposed to joint verification where the aggregate property $P_{1} \wedge \ldots \wedge P_{k}$ is used. Our approach is purely semantic, i.e., we do not rely on any structural features a design may or may not have.

We introduce a novel variant of separate verification called JA-verification. JA-verification checks if $P_{i}$ holds locally, i.e., under the assumption that all other properties are true. We show that if all properties hold locally, they also hold globally, i.e., without any assumptions. Instead of finding the set of all failed properties, JA-verification identifies a "debugging" subset. The properties in the debugging subset highlight design behaviors that need to be fixed first, which can yield substantial time savings in the design-verification cycle.

We experimentally compare conventional joint verification and JA-verification. We give examples of designs with failed properties where JA-verification dramatically outperforms its counterpart, especially for designs where a small debugging set $D$ exists. For these designs, one needs to find only $|D|$ CEXs, which are typically shallow. Computation of deeper CEXs for false properties that are not in $D$ is replaced with proving them true locally. Re-using inductive invariants generated for individual properties that are locally true significantly speeds up JA-verification. In particular, for correct designs, it makes JA-verification competitive with joint verification.

\section{REFERENCES}

[1] A. Biere, A. Cimatti, E. Clarke, M. Fujita, and Y. Zhu, "Symbolic model checking using SAT procedures instead of BDDs," in DAC, 1999, pp. 317-320.

[2] K. L. Mcmillan, "Interpolation and SAT-based model checking," in $C A V$. Springer, 2003, pp. 1-13.

[3] A. Bradley, "SAT-based model checking without unrolling," in VMCAI, 2011, pp. 70-87.

[4] N. Eén, A. Mishchenko, and R. Brayton, "Efficient implementation of property directed reachability," in FMCAD, 2011.

[5] E. Goldberg, M. Gudemann, D. Kroening, and R. Mukherjee, "Efficient verification of multi-property designs (the benefit of wrong assumptions) (extended version)," Tech. Rep. arXiv:1711.05698 [cs.LO], 2017.

[6] B. L. Synthesis and V. Group, "ABC: A system for sequential synthesis and verification," 2017, http://www.eecs.berkeley.edu/ alanmi/abc.

[7] H. Chockler, A. Ivrii, A. Matsliah, S. Moran, and Z. Nevo, "Incremental formal verification of hardware," in FMCAD, 2011.

[8] G. Cabodi and S. Nocco, "Optimized model checking of multiple properties," in DATE, 2011.

[9] Http://people.eecs.berkeley.edu/ alanmi/presentations/ updating_engines00.ppt.

[10] P. Camurati, D. P. C. Loiacono, P. Pasini, and S. Quer, "To split or to group: from divide-and-conquer to sub-task sharing in verifying multiple properties," in DIFTS, 2014.

[11] C. Jones, "Specification and design of (parallel) programs," in IFIP 9th World Congress, 1983, pp. 321-332.

[12] A. Pnueli, "In transition from global to modular temporal reasoning about programs," in Logic and Models of Conc. Sys., vol. 13, 1984, pp. 123-144. 